\title{
Avaliação da resistência coesiva de reparos de resina composta utilizando silano como agente de união
}

\author{
Evaluation of coesival resistance of resin repairs using silane as a coupling agent
}

Evaluación de la resistencia cohesiva de la reparación de resina compuesta con silano como agente de la unión

Ana Beatriz Cardoso Oliveira ${ }^{1 *}$, Matilde Verastegui Martins ${ }^{1}$, Alessandra Marangoni Fante ${ }^{1}$, Érika Matias Pinto Dinelly², Talita Arrais Daniel Mendes ${ }^{2,3}$, Marcelo Victor Sidou Lemos ${ }^{1,3}$.

\section{RESUMO}

Objetivo: Avaliar a resistência coesiva de reparos de resina composta utilizando silano como agente de união. Métodos: Foram confeccionados 48 blocos de resina compostas para dentina na cor A3,5 medindo $8 \times 8 \times 4$ $\mathrm{mm}$ que em sequência foram divididos em 4 grupos segundo a técnica de reparo: somente adesivo convencional; ácido fosfórico 37\%+adesivo convencional; ácido fosfórico 37\%+silano+adesivo convencional; ácido fosfórico a $37 \%$ + adesivo universal. Após isso, os blocos foram restaurados com resina composta translúcida, sendo 24 imediatamente após a confecção do bloco e 24 após conservação em água destilada por 24 horas. Os espécimes foram submetidos a teste de microtração antes após termociclagem. Os materiais utilizados para realização do reparo foram analisados em um espectrometro Micro-Raman. Resultados: Os grupos contendo silano apresentaram melhores resultados ao teste de microtração quando comparados aos grupos sem adição de silano $(P<0,05)$. No espectrofotômetro Micro-Raman o silano e o adesivo universal apresentaram picos aumentados de Si-O-Si quando comparados ao adesivo convencional. Conclusão: Pode-se concluir que a presença do silano interfere positivamente nos reparos de resinas compostas.

Palavras-chave: Adesivos dentinários, Falha de restauração dentária, Resinas compostas.

\begin{abstract}
Objective: To evaluate the coesival strength of resin repairs using silane as a bonding agent. Methods: 48 blocks of composite resin for dentin in color A3.5 measuring $8 \times 8 \times 4 \mathrm{~mm}$ were sequenced and divided into 4 groups, in which $\mathrm{G} 1=$ total-etch adhesive; $\mathrm{G} 2=37 \%$ phosphoric acid+conventional adhesive; $\mathrm{G} 3=$ Phosphoric acid 37\%+silane+total-etch adhesive; G4 $=37 \%$ phosphoric acid+universal adhesive. After that, the blocks were restored with a clear composite resin, 24 being immediately after the block was made and 24 after preservation in 24 hours distilled water. The specimens were submitted to microtensile test before and after artificial aging for 5000 cycles of thermocycling. The materials used in the study were analyzed in a MicroRaman spectroscopy. Results: The groups containing silane presented better results in the microtensile test when compared to the groups without silane addition $(P<0,05)$. In the Micro-Raman spectroscopy, the silane and the universal adhesive presented increased Si-O-Si peaks when compared to the total-etch adhesive. Conclusions: It can be concluded that the presence of silane interferes positively in repairs of composite resins.
\end{abstract}

Key words: Composite resins, Dental restoration failure, Dentin-bonding agents.

1 Universidade de Fortaleza - Fortaleza - CE - Brasil. *E-mail: talita_arrais@hotmail.com

${ }^{2}$ Centro Universitário Católica de Quixadá - Quixadá - CE- Brasil.

${ }^{3}$ Universidade Federal do Ceará - Fortaleza - CE- Brasil. 


\section{RESUMEN}

Objetivo: evaluar la resistencia cohesiva de las reparaciones de resinas compuestas utilizando silano como agente adhesivo. Métodos: se fabricaron cuarenta y ocho bloques de resina compuesta de dentina A3.5 de $8 \times 8 \times 4 \mathrm{~mm}$ y luego se dividieron en 4 grupos según la técnica de reparación: solo adhesivo convencional; $37 \%$ de ácido fosfórico + adhesivo convencional; $37 \%$ de ácido fosfórico + silano + adhesivo convencional; $37 \%$ de ácido fosfórico + adhesivo universal. Después de eso, los bloques se restauraron con resina compuesta translúcida, 24 inmediatamente después de que se hizo el bloque y 24 después de la conservación en agua destilada durante 24 horas. Las muestras se sometieron a pruebas microtensiles antes y después del envejecimiento artificial durante 5000 ciclos de termociclado. Los materiales utilizados para la reparación se analizaron utilizando un espectrofotómetro Micro-Raman. Resultados: Los grupos que contienen silano presentaron mejores resultados para la prueba de microtensil en comparación con los grupos sin adición de silano $(P<0,05)$. En el espectrofotómetro Micro-Raman, el silano y el adhesivo universal presentaron picos de Si-O-Si aumentados en comparación con el adhesivo convencional. Conclusiones: la presencia de silano interfiere positivamente con las reparaciones de resina compuesta.

Palabras clave: Adhesivos de dentina, Falla de restauración dental, Resinas compuestas.

\section{INTRODUÇÃO}

Com o passar do tempo a exigência estética da população resultou no maior uso da resina composta em relação a outros materiais restauradores, trazendo como consequência a melhoria das propriedades mecânicas deste material ao longo dos anos. As resinas compostas visam copiar características naturais do dente como cor, textura e translucidez (SILVA TM, 2019).

As resinas compostas têm em sua composição uma matriz orgânica, inorgânica e um agente de união, além de sistemas ativadores e inibidores de fotopolimerização. A matriz orgânica é geralmente formada por monômeros de alto ou baixo peso molecular, como o BIS-GMA, TEGDMA, UDMA, entre outros. A matriz inorgânica é formada por partículas de carga, que empregam função essencial nas propriedades mecânicas das resinas compostas. O agente de união, no qual é comumente utilizado o silano, também tem sua função primordial na composição da resina composta, sendo responsável pela união da matriz orgânica à inorgânica, permitindo a distribuição de estresse por todo o material e evitando uma fonte primária de fratura (RIBEIRO JCR, 2008; ANUSAVICE KJ, et al., 2013).

Apesar de ter como propriedade uma boa vida útil (IRMAK Ö, et al., 2016), com o passar do tempo, esses compósitos podem apresentar algumas falhas como, por exemplo, alterações de cor, fraturas (PAPACCHINI F, et al., 2007), fendas marginais e cáries secundárias (IRMAK Ö, et al., 2016) ao redor das margens ou até mesmo na face proximal oposta à restauração (CHRISTENSEN GJ, 2007). Com isso, algumas propostas foram formuladas com o intuito de melhorar a composição das resinas compostas e técnicas clínicas foram aprimoradas pelos dentistas (ALTINCI P, et al., 2017).

A substituição da restauração e a reparação do defeito são exemplos de métodos utilizados, onde o primeiro é considerado bastante invasivo, pois poderia haver a remoção de tecido dental sadio (SARTORI N, et al., 2010), levando a restaurações maiores e mais complexas, como também a uma provável terapia de canais radiculares ou até mesmo perda do dente (IRMAK Ö, et al., 2017). Por sua vez, o reparo seria o método mais indicado por ser mais conservador ao manter o tecido sadio intacto (SARTORI N, et al., 2010) ter melhor custo-benefício e menor tempo clínico (PAPACCHINI F, et al., 2007). Porém, a escolha do método adequado vai depender de fatores como: presença de microinfiltrações, incompatibilidade da cor da restauração com a estrutura dentária e presença de cárie secundária (SARTORI N, et al., 2010).

Para a reparação do defeito seria indicado um preparo da superfície que vai receber o material novo utilizando ácido fosfórico, jateamento com óxido de alumínio ou uso de pontas diamantadas para manter a superfície áspera e remover a camada superficial alterada pela exposição à saliva, aumentando a área e 
energia de superfície da resina composta, além de aplicação de agentes de acoplamento, como o silano (IRMAK Ö, et al., 2017).

O silano presente nas resinas compostas possui a capacidade de união da matriz inorgânica dessas com a matriz orgânicas, quando presente em adesivos aumenta o escoamento nas superfícies irregulares sendo considerado importante para a otimizar a resistência de união dos reparos nessa superfície (BACCHI A, et al., 2010). Tanto para a reparação, quanto para a substituição, os sistemas adesivos são materiais imprescindíveis quando se fala em restaurações com resina composta, pois há um aumento significativo na qualidade da reparação quando são utilizados. Os sistemas adesivos são divididos em dois grupos: autocondicionantes e os de condicionamento total (IRMAK Ö, et al., 2016).

Diante da constante evolução dos materiais dentários restauradores, uma nova tendência vem sendo os adesivos universais que são muito mais versáteis, pois podem ser aplicados por meio de diferentes técnicas, como condicionamento total e autocondicionantes, além de técnicas de ataque seletivo. Eles têm a capacidade de unir vários tipos de substratos, como: vidro, cerâmica, zircônia, ligas de metais, além de remanescentes dentários (SUDA S, et al., 2018).

Alguns adesivos universais já apresentam o silano como agente de união em suas composições, podendo ser visto como uma alternativa quando falamos em reparos de resina composta. Porém, nenhum artigo prévio comparou a efetividade da utilização do agente de união (silano) separadamente ou incorporado à sistemas adesivos universais. Logo, o objetivo do presente trabalho é avaliar se a presença do silano, incorporado ou não ao sistema adesivo, contribui de forma efetiva na resistência de união do reparo de resinas compostas, tendo como justificativa o custo benefício para o paciente, impedindo desnecessário desgaste do elemento dentário e menor tempo clínico ao cirurgião dentista, e como hipótese temos que a presença do silano interfere positivamente nos reparos de resina composta.

\section{MÉTODOS}

\section{Delineamento experimental}

Os fatores de estudo sob investigação foram o uso de diferentes técnicas para reparo de resinas compostas, sendo divididos em quatro níveis: (1) aplicação do adesivo convencional, seguido de fotoativação; (2) aplicação de ácido fosfórico 37\%, seguido de adesivo convencional e fotoativação; (3) aplicação de ácido fosfórico $37 \%$, seguido de silano, adesivo convencional e fotoativação e (4) aplicação de ácido fosfórico $37 \%$, seguido de adesivo universal contendo silano e fotoativação.

As variáveis dependentes do estudo foram: resistência coesiva, medida em uma máquina de ensaios universais; e padrão de fratura, analisado em uma lupa estereoscópica. Para cada grupo experimental foram utilizados 6 espécimes, os quais foram analisados de forma imediata e após 5000 ciclos térmicos. Para análise qualitativa, os materiais utilizados para realizar reparo foram submetidos a espectroscopia micro-Raman.

\section{Preparo dos blocos de resina composta}

Foram confeccionados 48 blocos de resinas composta nanohíbridas (Opallis FGM, Joinville, SC, Brasil). A confecção dos blocos constituiu-se de inserção de dois incrementos de $2 \mathrm{~mm}$ de resina $A 3,5$ para dentina em uma matriz de teflon, resultando em blocos de $8 \times 8 \times 4 \mathrm{~mm}$. Após a última polimerização os espécimes foram armazenados em ambiente úmido por 30 minutos ( 24 blocos) e 24 horas ( 24 blocos).

Posteriormente, a face oposta a matriz de teflon foi lixada manualmente em lixa de carbeto de silício de granulação 600 durante 30 segundos sob refrigeração constante. Em seguida, os espécimes foram secos com papel absorvente e jatos de ar e foram aplicados os devidos tratamentos de superfície:

G1 - Aplicação do adesivo (Single Bond 2, 3M ESPE, CA, EUA), evaporação do solvente durante 10 segundos com jato de ar a $30 \mathrm{~cm}$ da amostra e fotoativação por 40 segundos.

G2 - Aplicação de ácido fosfórico a 37\% (Maquira, Maringá, PR, Brasil) por 15 segundos, lavagem com água destilada corrente por 30 segundos, secagem com papel absorvente e jatos de ar, aplicação do adesivo 
(Single Bond 2, 3M ESPE, CA, EUA), evaporação do solvente durante 10 segundos com jato de ar a $30 \mathrm{~cm}$ da amostra e fotoativação por 40 segundos.

G3 - Aplicação de ácido fosfórico a 37\% (Maquira, Maringá, PR, Brasil) por 15 segundos, lavagem com água destilada corrente por 30 segundos, secagem com papel absorvente e jatos de ar, aplicação ativa de silano (Prosil FGM, Joinville, SC, Brasil) por 60 segundos, seguido de secagem com jato de ar por 10 segundos, aplicação do adesivo (Single Bond 2, 3M ESPE, CA, EUA), evaporação do solvente durante 10 segundos com jato de ar a $30 \mathrm{~cm}$ da amostra e fotoativação por 40 segundos.

G4 - Aplicação de ácido fosfórico a 37\% (Maquira, Maringá, PR, Brasil) por 15 segundos, lavagem com água destilada corrente por 30 segundos, secagem com papel absorvente e jatos de ar, aplicação do adesivo universal contendo silano (Single Bond Universal, 3M ESPE, CA, EUA), evaporação do solvente durante 10 segundos com jato de ar a $30 \mathrm{~cm}$ da mostra e fotoativação por 40 segundos.

Após tratamento de superfície e armazenamento, os espécimes foram restaurados com dois incrementos de $2 \mathrm{~mm}$ de resina translúcida (T-neutral Opallis FGM, Joinville, SC, Brasil), resultando em blocos de $64 \mathrm{~mm}^{2}$ e $8 \mathrm{~mm}$ de altura. Os blocos reparados foram armazenados durante 24 horas em ambiente úmido para posterior confecção dos espécimes a serem testados.

\section{Composição dos materiais}

Quadro 1 - Composição dos materiais utilizados na pesquisa.

\begin{tabular}{|l|l|l|l|}
\hline \multicolumn{1}{|c|}{ Material } & Fabricante & UF/País & \multicolumn{1}{c|}{ Composição } \\
\hline Single Bond 2 & 3M ESPE & CA, EUA & $\begin{array}{l}\text { BisGMA, HEMA, dimetacrilatos, etanol, água, um inovador } \\
\text { sistema fotoiniciador e um copolímero funcional de metacrilato } \\
\text { de ácidos poliacrílico e polialcenóico e nanopartículas de sílica }\end{array}$ \\
\hline $\begin{array}{l}\text { Single Bond } \\
\text { Universal }\end{array}$ & 3 M ESPE & CA, EUA & $\begin{array}{l}\text { Monômero de fostato de MDP, resinas de dimetacrilato, } \\
\text { copolímero Vitrebond TM, enchedor, etanol, água, iniciadores } \\
\text { e silano }\end{array}$ \\
\hline Prosil & FGM & SC, BRA & $\begin{array}{l}\text { 3-Metacriloxipropiltrimetoxisilano <5\%, etanol > 85\%, Água }< \\
10 \%\end{array}$ \\
\hline Opallis & FGM & PR, BRA & $\begin{array}{l}\text { Ingredientes ativos como monômeros de Bis-GMA (Bis-Fenol } \\
\text { A di-Glicidil Metacrilato), BisEMA (BisFenol A di-Glicidil } \\
\text { Metacrilato toxilado), TEGDMA (Trietileno glicol } \\
\text { dimetacrilato), UDMA (Uretano dimetacrilato), canforquinona, } \\
\text { co-iniciador e silano e ingredientes inativos como vidro de } \\
\text { bário-alumino silicato silanizado, pigmentos e sílicas. }\end{array}$ \\
\hline
\end{tabular}

Fonte: Oliveira ABC, et al., 2019.

\section{Teste de Resistência Coesiva}

Os blocos foram cortados em uma cortadeira metalográfica de precisão em barras de 1,7x1x6mm, sob refrigeração constante. Posteriormente foram realizados entalhes com uma ponta diamantada esférica 1014 em ambos os lados da interface de transição entre as resinas, a fim de criar um espécime em formato de ampulheta. Metade dos espécimes foram testados de forma imediata e o restante foi submetido a ciclagem térmica para simulação de envelhecimento.

As amostras foram colocadas em dispositivo de Geraldeli com cola de cianoacrilato (Super Bonder, Loctite Ltda, São Paulo, Brasil) e submetidos a uma máquina de ensaios universais (DL2000, EMIC, Canton, MA, USA) com uma célula de carga de $500 \mathrm{~N}$ e velocidade de $0,5 \mathrm{~mm}$ por minuto a fim da avaliação a resistência coesiva dos espécimes. Após a fratura, a dimensão da área fraturada foi mensurada com um paquímetro digital e a resistência de união em megapascal (MPa) foi calculada. 


\section{Ciclagem Térmica}

Os blocos foram envelhecidos artificialmente (TC45, SD Mechatronik, Feldkirchen-Westerham, ALE) por meio de 5000 ciclos de termociclagem a uma temperatura de $5^{\circ} \mathrm{C}$ e $55^{\circ} \mathrm{C}$ com 20 segundos em banho e 20 segundos de transferência. Após isso, realização do envelhecimento, as amostras foram submetidas ao teste de resistência coesiva descrito anteriormente.

\section{Espectroscopia Micro-Raman}

Os agentes utilizados para promover adesão durante o reparo foram analisados em espectrômetro Raman (Xplora, Horiba, Paris, França) acoplado a um microscópio óptico. Para tanto, uma gota de a gente foi aplicada sobre uma lâmina de vidro, posteriormente foram analisados com laser $532 \mathrm{~nm}^{-1}$, potência de $0,1 \%$, com espectro de avaliação entre 400-2000. Após aquisição os gráficos foram submetidos a realização de baseline e eliminação de ruídos de forma padronizada.

\section{Análise estatística}

Para análise estatística cada espécime em ampulheta foi considerado como valor independente, resultando em 24 espécimes para cada grupo testado $(n=24)$. Os dados foram submetidos a um teste de normalidade de Kolmogov-Smirnov e posteriormente analisados por ANOVA a dois critérios, seguido de pósteste de Tukey. Em todas as situações adotou-se nível de significância de 5\% $(p<0,05)$.

\section{RESULTADOS}

Quando analisados os valores de resistência coesiva após 30 min de espera para a realização do reparo (Tabela 1), podemos notar que o grupo tratado com silano não incorporado se mostrou superior aos demais grupos quando testado imediatamente. Já após ciclagem, mostrou-se semelhante ao grupo tratado com adesivo universal $(p=0,054)$, porém superior aos demais grupos.

Tabela 1 - Valores de resistência coesiva (desvio-padrão) expressos em MPa após 30 minutos de espera para realização do reparo.

\begin{tabular}{ccccc}
\hline & $\mathbf{A C}$ & $\mathbf{A}+\mathbf{A C}$ & $\mathbf{A}+\mathbf{A U}$ & $\mathbf{A}+\mathbf{S I L}+\mathbf{A C}$ \\
\hline Imediato & $30,8(6,4) \mathrm{Ba}$ & $30,2(8,2) \mathrm{Ba}$ & $33,2(9,4) \mathrm{Ba}$ & $44,3(7,2) \mathrm{Aa}$ \\
Ciclado & $29,8(6,7) \mathrm{Ba}$ & $32,1(6,9) \mathrm{Ba}$ & $33,0(7,9) \mathrm{ABa}$ & $40,7(8,1) \mathrm{Aa}$ \\
\hline
\end{tabular}

Legenda: Letras maiúsculas indicam diferenças estatísticas em comparação de linha. Letras minúsculas indicam diferenças estatísticas em comparação de coluna. Os grupos A indica condicionamento ácido; AC indica adesivo convencional; AU indica adesivo universal e SIL indica silano.

Fonte: Oliveira ABC, et al., 2019.

Quando analisados os valores de resistência coesiva após 24 horas de espera para realização do reparo (Tabela 2), podemos observar que de forma imediata o grupo tratado com adesivos universais apresentou resultados aumentados em comparação aos demais grupos. Por sua vez, após ciclagem, mostrou-se semelhante ao grupo com silano não incorporado $(p=0,523)$, embora superior aos demais grupos.

Tabela 2- Valores de resistência coesiva (desvio-padrão) expressos em MPa após 24 horas de espera para realização do reparo.

\begin{tabular}{ccccc}
\hline & AC & A + AC & A + AU & A + SIL + AC \\
\hline Imediato & $37,1(5,8) \mathrm{Ba}$ & $34,6(7,3) \mathrm{Ba}$ & $50,1(7,0) \mathrm{Aa}$ & $41,6(7,5) \mathrm{Ba}$ \\
Ciclado & $32,7(6,0) \mathrm{Ba}$ & $32,2(7,1) \mathrm{Ba}$ & $40,9(5,8) \mathrm{Ab}$ & $37,5(6,2) \mathrm{ABa}$ \\
\hline
\end{tabular}

Legenda: Letras maiúsculas indicam diferenças estatísticas em comparação de linha. Letras minúsculas indicam diferenças estatísticas em comparação de coluna. Os grupos A indica condicionamento ácido; AC indica adesivo convencional; AU indica adesivo universal e SIL indica silano.

Fonte: Oliveira ABC, et al., 2019. 
Quando analisados os espectros dos diferentes agentes utilizados para promover a união entre resina opaca e resina translúcida, podemos verificar que o silano e o adesivo universal apresentaram picos referentes a ligação Si-O-Si (450-550 $\mathrm{nm}^{-1}$ ) aumentados em relação ao adesivo convencional, demonstrando uma maior disponibilidade de silano para realização de ligações (Gráfico 1).

Gráfico 1 - Espectros micro-Raman dos materiais utilizados para reparo em resina composta.

A

Silano (Prosil, FGM)

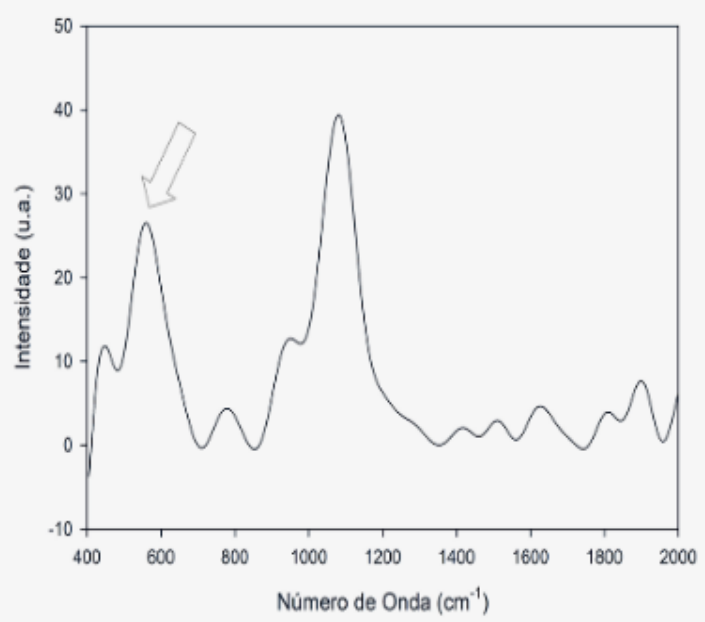

B

Adesivo Universal (Single Bond Universal, 3M ESPE)

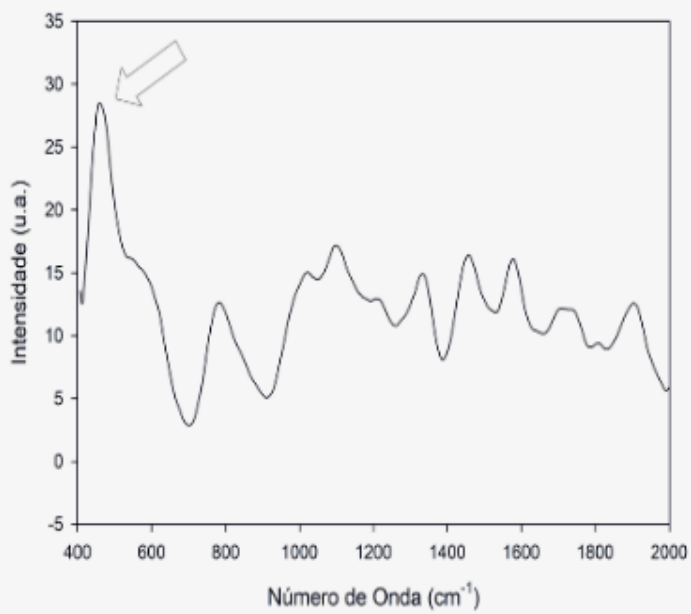

C Adesivo Convencional (Single Bond 2, 3M ESPE)

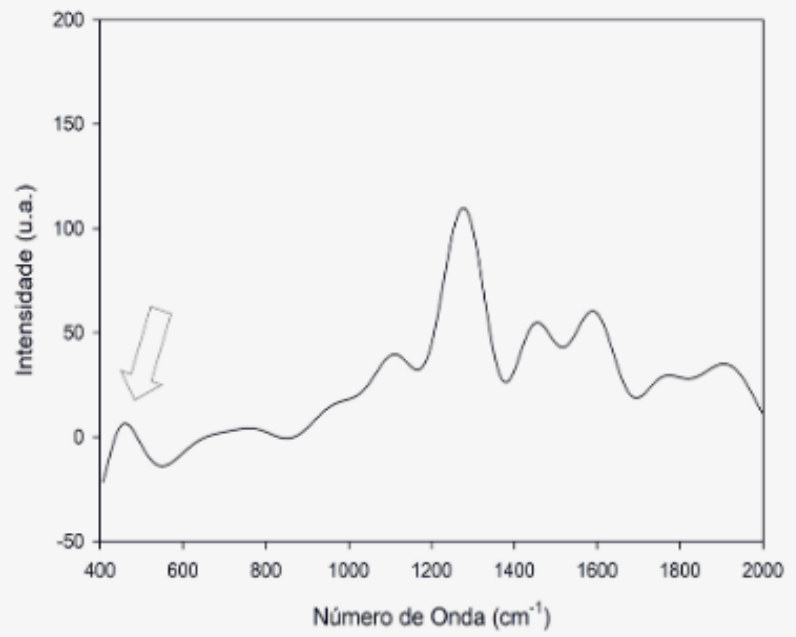

Legenda: Silano (Prosil, FGM) (A), adesivo universal (Single Bond Universal, 3M ESPE) (B) e adesivo convencional (Single Bond 2, 3M ESPE) (C). As setas abertas indicam picos de ligação Si-O-Si (450-550 nm-1). Fonte: Oliveira ABC, et al., 2019.

\section{DISCUSSÃO}

Essa pesquisa teve como relevância esclarecer ao cirurgião dentista sobre formas de reparo em resina composta, mostrando como diferentes sistemas adesivos se portam nessa situação. Diferente da maioria dos estudos encontrados na literatura (IRMAK Ö, et al., 2017; ALTINCI P, et al., 2017; SARTORI N, et al., 2010) o presente estudo optou pela utilização da realização de um teste de resistência coesiva, tendo em vista que 
os materiais possuem composição semelhante e existe uma tendência para falhas em defeitos existentes na confecção dos platôs de resina. Tal escolha se deu a partir da teoria de Griffith, que propõe que quando existe uma trinca em um material, a energia passa a se concentrar na localidade da fenda, culminando na fratura do espécime (FUJITA H, et al., 2006).

Optou-se também pela realização de dois fatores de teste, o primeiro reparando o bloco de resina de forma imediata e o segundo reparando depois de colocado por 24 horas em água destilada. Tal escolha se deu ao fato de que a conversão de monômeros em polímeros da resina composta se completa após 24 horas, apesar de previamente fotoativada. Assim foi verificado se tais monômeros ainda em processo de polimerização poderiam influenciar no processo de reparação, fato que não pode ser confirmado pelo presente estudo.

Os sistemas adesivos universais apareceram no mercado com o intuito de simplificar a prática clínica diária e diminuir o custo do cirurgião dentista, oferecendo em um só produto os sistemas adesivos autocondicionante e condicionamento total, prometendo também eficácia quando usado em substratos diferentes do dente, como resinas compostas, metais, zircônia e cerâmicas a base de sílica. De acordo com fabricantes de sistemas adesivos universais, o material tem como indicação o uso em restaurações diretas e indiretas nos diferentes sistemas adesivos, primer em zircônia, metal e alumina, além de substituir o silano em restaurações indiretas (Oz FD, et al., 2019).

A presente pesquisa não demonstrou diferença estatística após 5000 ciclos de termociclagem, concordando com o estudo de Kiomarsi N (2017) que realizou um estudo com 5000 e 10000 ciclos. Em contrapartida, quando se optou por 10000 ciclos de termociclagem os resultados mostraram que interferiu negativamente por ocasionar degradação hidrolítica (KIOMARSI N, et al., 2017). O fato de a presença do silano ter agido positivamente nos reparos de resina composta pode ser justificado pelo fato de que o silano pode ser capaz de aumentar o escoamento do sistema adesivo nas superfícies irregulares (IRMAK Ö, et al., 2016).

O fato de o silano usado separadamente ter apresentado melhores resultados no reparo imediato e o adesivo universal contendo silano em sua composição ter se portado melhor no reparo após 24 horas concorda com o estudo de Staxrud F e Dahl JE (2015), que relataram que a aplicação de silano antes do sistema adesivo ou o uso de um sistema adesivo contendo silano na composição aumentou a resistência de união da resina composta envelhecida. Tal fato nos faz constatar que o material por si só interfere de forma positiva nos reparos de resina composta, independentemente de sua forma de apresentação. Por esse motivo, a hipótese inicial do presente estudo foi confirmada (STAXRUD F, DAHL JE, 2015).

Segundo a literatura picos fortes entre $450-550 \mathrm{~nm}^{-1}$ indicam ligações do tipo $\mathrm{Si}-\mathrm{O}-\mathrm{Si}$, sendo estas ligações abundantes na molécula de silano (SUDA S, et al., 2018). Logo o silano e o adesivo universal testado apresentaram picos aumentados nas referidas regiões em relação ao adesivo convencional, fortalecendo a hipótese de que o silano se apresenta como fator determinante para um reparo com boa resistência coesiva.

O estudo de Neves e colaboradores (NEVES TPC, et al., 2017) nos mostrou que os valores em Mpa de um teste de microtração analisando a resistência de união entre a dentina e um adesivo convencional de dois passos (Single Bond 2) não se mostraram superiores aos valores dos resultados deste estudo. Desta forma é possível afirmar que o reparo de resina composta é uma alternativa diante de fraturas de restaurações, nem sempre precisando removê-las por completo (NEVES TPC, et al., 2017).

\section{CONCLUSÃO}

O estudo confirmou a hipótese de que esse material agiu de forma positiva no reparo de restaurações nas duas formas analisadas, expondo ao cirurgião-dentista o adesivo universal como forma alternativa de sistema adesivo que, apesar de simplificado, apresentou bons resultados quando submetido ao teste de microtração. O presente estudo limitou-se ao fato de ser um estudo laboratorial utilizando apenas dois tipos de sistemas adesivos e com ciclagem térmica com período reduzido. Sugere-se estudos complementares utilizando sistemas adesivos diversos com e sem adição de silano, bem como o teste de diferentes marcas de adesivos universais. 


\section{AGRADECIMENTOS E FINANCIAMENTO}

À Coordenação de Aperfeiçoamento Pessoal de Nível Superior. Ao Programa de Pós-Graduação em Odontologia da Universidade Federal do Ceará.

\section{REFERÊNCIAS}

1. CHRISTENSEN GJ. When and how to repair a failing restoration. The Journal of The American Dental Association, 2007; 138(12): 1605-1607.

2. STAXRUD F, DAHL JE. Silanising agents promote resin-composite repair. Int Dent J. 2015 dez; 65 (6): $311-315$.

3. ALTINCI P, et al. Repair bond strength of nanohybrid composite resins with a universal adhesive. Acta Biomaterialia Odontologica Scandinavica, 2017; 4(1): 10-19.

4. BACCHI A, et al. Reparos em restaurações de resina composta - revisão de literatura. Revista da Faculdade de Odontologia, 2010; 15(3): 331-335.

5. FUJITA H, et al. Contribuição do estudo da retificação abrasiva de materiais cerâmicos. Cerâmica. 2006 dez; 52 (324): 269-275.

6. IRMAK Ö, et al. Adhesive system affects repair bond strength of resin composite. Journal of Istanbul University Faculty Of Dentistry, 2017; 51(3): 25-31.

7. IRMAK Ö, et al. Solvent type influences bond strength to air or blot-dried dentin. Bmc Oral Health, 2016; 16(1): 30-35.

8. KIOMARSI N, et al. Effect of thermocycling and surface treatment on repair bond strength of composite. $\mathrm{J}$ Clin Exp Dent. 2017; 9 (8): 945-951.

9. NEVES TPC, et al. Resistência de união à microtração de sistemas adesivos "condiciona-e-lava" de dois passos: efeito de diferentes tratamentos da superfície dentinária condicionada. Rev Odontol UNESP. 2017 mai-jun; 46 (3): 131-137.

10. OZ FD, et al. Twenty-four-month clinical performance of different universal adhesives in etch-and-rinse, selective etching and self-etch application modes in NCCL - a randomized controlled clinical trial. J Appl Oral Sci. 2019 abr; 27: 1-10.

11. PAPACCHINI F, et al. Hydrolytic stability of composite repair bond. European Journal of Oral Sciences, 2007; 115(5): 417-424.

12. RIBEIRO JCR, et al. Shear strength evaluation of composite-composite resin associations. Journal of Dentistry, 2008; 36(5): 326-330.

13. SARTORI N, et al. Avaliação da resistência à tração de reparos de restaurações de resina composta. Arquivos em Odontologia, 2010; 46(4): 190-196.

14. SILVA TM, et al. Surface degradation of composite resins under staining and brushing challenges. Journal of Dental Sciences, 2019; 14(1): 87-92.

15. SUDA S, et al. Comparison of enamel bond fatigue durability between universal adhesives and two-step self-etch adhesives: Effect of phosphoric acid pre-etching. Dental Materials Journal, 2018; 37(2): 244-255.

16. ANUSAVICE KJ, SHEN C, RAWLS HR. Phillips Materiais Dentários. 12. ed. Rio de Janeiro: Elsevier, 2013; 592p. 